\title{
A Plant-enriched Diet and Long-term Health, Particularly in Reference to China
}

\author{
T. Colin Campbell \\ Division of Nutritional Sciences, Cornell University, Ithaca, NY 14853
}

The nutritional value of fruits, vegetables and cereal products has been emphasized in several pronouncements on dietary recom mendations designed to avoid chronic, degenerative diseases (e.g., cardiovascular disease and cancers). These diseases are common in Western industrialized countries and have begun to emerge in developing countries such as China.

A brief review of the nutritional etiology of these diseases, as existing in China. suggests that an increase in the consumption of total dietary fat and animal protein. along with other nutrients correlated with these changes, chiefly accounts for this epidemiological transition. This nutritional relationship. when combined with the enormous relative cost of producing animal products, suggests that developing counties should avoid putting emphasis on the produc tion of livestock and instead focus on increasing the availability and improving the quality of foods of plant origin, especially fruits and vegetables.

Dietary guidelines to increase the consumption of a variety of foods of plant origin in industrialized countries therefore would be consistent with food and nutrition policies in developing countries.

A number of dietary recommendations have been promoted in recent years by various government agencies and public health organizations. Nearly all are directed to ways in which chronic degenerative diseases, such as cardiovascular disease and various cancers for example, might be prevented. These diseases. along with diabetes, are responsible for the vast majority of deaths in the United States and other Western industrialized countries.

Dietary guidelines designed for prevention of these diseases generally recommend consumption of less dietary fat. especially saturated fat, and more dietary fiber and foods rich in the antioxidant vitamin family. such as the carotenoids, vitamin E, and vitamin C (O'Connor and Campbell, 1986). More to the point of this conference, however. is the recommendation to consume more vegetables. cereals, and fruits. The nutritional justification and implications of this latter guideline is discussed here.

Recommendations that advocate "more" or "less" consumption refer, of course. to the average American diet. One way to obtain a comprehensive picture of the American diet is to compare the proportion of nutrient intake coming from foods of animal origin vs. the proportion coming from foods of plant origin. Nutrient intakes that may be used to illustrate this type of dietary division are protein and calcium, because their consumption mostly comes from foods rather than from nutrient supplements or nutrient-rich extracts. Also, these two nutrients are present in abundance in some foods of each of these two food groups. Incidentally, it is more appropriate to use protein or calcium rather than energy to indicate the proportions of plant and animal foods in the diet because so much of the energy intake comes from extract-rich oils and fats used in food preparation and presentation.

Comparative intakes of protein and calcium show that. on average, about three-fourths of the food consumed in the United States comes from foods of animal origin. including meats, dairy produce, eggs. and their various by-products. What is especially remarkable about this observation is the notion that about half of all Americans actually consume an even higher proportion of these foods.

Mean nutrient intakes in the United States can be described more fully by comparing the intakes of other nutrients with those of rural China, where the consumption of animal products by most people has been much lower. Shown in Table 1 are comparisons available in the study by Chen et al. (1990). Fat intake is far higher in the United States, although it is now increasing quite rapidly in certain urban areas of China (Gladstone Foundation, 1983). Average dietary fiber intake in China, at $33 \mathrm{~g} /$ day, is about three times higher than is the intake in the United States and can be. as a county mean, as high as $77 \mathrm{~g} / \mathrm{day}$. The much higher level of intake of soluble carbohydrates in China is attributable to a high level of consumption of cereal grains, and. in some areas. tubers. The far lower level of intake of calcium in China would appear to predispose them to the risk of osteoporosis. but, on the basis of anecdotal evidence. this seems not to be true. In any case, we are presently investigating this question in another survey in greater depth. Total protein intake in the United States is $40 \%$ higher, but, of greater consequence. the mean percent of total protein as animal protein in the United States is $70 \%$. whereas in China it is only $7 \%$. Mean iron intake is almost twice as high in China, and contrary to the im pressions of some, anemia, at least in adults. was not observed. although almost all of the iron comes from plant sources: Thiamin intake in China, like soluble carbohydrates, reflects the greater consumption of cereals. The intake of retinol (vitamin A), exclusively found in animal products, is far higher in the United States. Also note that average energy intake in China is actually higher than the 
Table 1. Comparison of American diet with rural Chinese diet (mean daily intakes).

\begin{tabular}{llc}
\hline Intake & China & United States \\
\hline Total fat (\% of kcal) & $38-40$ & 15 \\
Dietary fiber (g/day) & $10-12$ & 33 \\
Soluble carbohydrate (g/day) & 240 & 470 \\
Calcium (mg/day) & 1140 & 540 \\
Protein (g/day, 70-kg male) & $90-95$ & 64 \\
Animal protein (\% of total) & 70 & 7 \\
Iron (mg/day) & 18 & 34 \\
Thiamin (mg/day) & 1.4 & 2.3 \\
Retinol (RE/day) & 990 & 30 \\
Energy intake (kcal/day) & 2360 & 2640 \\
\hline- & &
\end{tabular}

intakes of comparable individuals in the United States and. in spite of this, mean body mass index is $25 \%$ higher in the United States.

How do average Chinese and American diets compare with American dietary recommendations? About the only quantitative recommendations available for this comparison are those for total fat and dietary fiber. Recommended levels for dietary fat are $30 \%$ or less (National Academy of Sciences, 1989) and for dietary fiber are 20 to $35 \mathrm{~g} /$ day (Federation of American Societies for Experimental Biology. 1987). These comparisons of recommended and actual intakes of fat and fiber make it quite clear that what is being recommended for future consumption in the United States is something approaching the Chinese experience.

However, when interpreting these comparisons, it also is impor tant to note for the Chinese diet a second feature related to food choice; namely, the Chinese consume very simple and monotonous diets. Even though they consume great quantities, rural Chinese use far fewer plant foods than Americans-as a stroll through an American supermarket readily indicates. The average number of plant foods consumed at the time of the survey in each county was nine to 10 , with the addition of another one to two animal foods. In the United States, $\approx 90 \%$ and $95 \%$ by weight of the average diet is comprised of 500 and 900 different kinds of food, respectively (Pennington, 1983). Consumption of plant foods in China is limited mostly to those that are in season, although in some areas significant amounts vegetables and fruits preserved by nonrefrigeration procedures of salting or fermentation also are consumed. So, even though Americans consume much less plant food in terms of quan tity, there is much more variety; moreover, there is better quality (i.e., more refrigerated and sterilized foods) and there is significant consumption of fresh, uncooked fruits and vegetables. On the ques tion of variety, it is important to consume a mixture of plant foods, primarily to be assured of adequate intakes of all nutrients, many of which are enriched in some plant foods but not in others.

Are dietary guidelines of the United States relevant for developing countries? This question can be considered at two levels of evi dence. using a comparison of Chinese and American dietary and health characteristics as the evidence. First, the question is consid ered only in reference to the dietary guidelines as published. That is. if it is assumed that these guidelines are based on convincing ev idence, then I would suggest. for Chinese and other peoples in simi lar dietary situations. that it would be in their long-term health inter ests not to stray too far from their present balance of nutrient intake. This is best achieved by not putting unnecessary emphasis on the development of a livestock industry, either for the production of meat. dairy products, or eggs. I am not suggesting that these foods be avoided, but rather that there is little or no evidence stated in support of Western dietary guidelines that animal food products contain anything of value for prevention of chronic degenerative diseases. A future increase in the consumption of animal food products would cause a shift in dietary practices opposite to those being advocated by these guidelines. Instead, for countries like China. the chief emphasis for future food and nutrition policies should be centered on the need for variety in the consumption of plant produce, not on the need for increased consumption of foods of animal origin. This emphasis on variety in the consumption of plant foods directs attention in turn to the need for improving proce dures for the transport and storage of foods so that they can be dis tributed beyond local production areas and be saved over different seasons. particularly for fruits and vegetables.

The second level of evidence determining the relevance of Western dietary guidelines for developing countries is to examine the supporting evidence for these guidelines, particularly if the evidence is obtained in a developing country. For this analysis, the data of China are used (Chen et al.. 1990).

A first approximation of the value of plant foods towards the prevention of Western-type diseases in China can be obtained by examination of the associations between the consumption of green vegetables and legumes and various age-standardized mortality rates (Table 2). The statistically significant association with coronary heart disease may be quite remarkable when we take note of the fact that this disease is much less common in China than in the United States. In other words, with these low mortality rates, there is less sensitivity for detection of a statistically significant association. Thus, when one is detected, as is the case here, the result is quite remarkable. On the basis of international comparisons, mor tality from coronary heart disease may be lower in China, in part, because of the relatively high consumption of green vegetables. The protective effect of green vegetables on cancer mortality rates, especially on total aggregate cancer, also is predicted by the evidence supporting Western dietary guidelines. Similar implications are drawn for the consumption of legumes.

Table. 2. Protective associations of consumption of various plant foods with various mortality rates in China.

\begin{tabular}{|c|c|}
\hline $\begin{array}{l}\text { Frequency of } \\
\text { vegetable consumption }\end{array}$ & $\begin{array}{l}\text { vs. Coronary heart disease** } \\
\text { Hypertensive heart disease* } \\
\text { Stroke* } \\
\text { Total cancer* } \\
\text { Esophageal cancer* } \\
\text { Stomach cancer** } \\
\text { Cervical cancer** }\end{array}$ \\
\hline $\begin{array}{l}\text { Daily legume } \\
\text { consumption }\end{array}$ & $\begin{array}{l}\text { vs. Cervical cancer* } \\
\text { Hypertensive heart disease* } \\
\text { Stroke* }\end{array}$ \\
\hline
\end{tabular}

$*, * * \mathrm{P}<0.05$ or 0.01 , respectively.

Table 3. Comparison of metabolic/clinical parameters in the United States and China.

\begin{tabular}{|c|c|c|}
\hline Parameter & United States & China $^{2}$ \\
\hline Total cholesterol (mg/dl) & $127^{y}$ & 212 \\
\hline LDL cholesterol (mg/dl) & 85 & 148 \\
\hline Total cholesterol/HDL cholesterol & 2.91 & 4.24 \\
\hline Plasma tiglycerides (mg/dl) & 97 & 120 \\
\hline Plasma albumin $(\mathrm{g} / \mathrm{dl})$ & $3.0^{x}$ & 4.4 \\
\hline Plasma urea nitrogen (mg/dl) & $10^{w}$ & 12.5 \\
\hline Plasma beta-carotene $(\mu \mathrm{g} / \mathrm{dl})$ & 17 & 35 \\
\hline Height $(\mathrm{cm}) 164$ & 173 & \\
\hline Weight (kg) 55 & 77 & \\
\hline Body mass index (weight/height ${ }^{2}$ ) & 20.5 & 25 \\
\hline
\end{tabular}

$\bar{z}$, "Reference male" in China (65kg adult male of light physical labor) and comparable individual in the United States. Most of these data are comparisons of means.

${ }^{\mathrm{y}}$ Correlated with total fat intake $(P<0.01)$ and meat intake $(\mathrm{P}<0.01)$.

${ }^{\mathrm{x}}$ Correlated with coronary heart disease $(\mathrm{P}$ c 0.01) and total cancer $(\mathrm{P}<$ $0.001)$.

${ }^{\text {w }}$ Correlated with childhood total cancer $(\mathrm{P}<0.01)$ and animal protein intake ( $\mathrm{P}<0.001)$. 
The relationship between dietary practices and Western-type diseases in China also can be considered from another perspective, namely, the associations between these disease mortality rates and metabolic indicators of nutritional status (Table 3). Total plasma cholesterol is much lower in rural China, with a range of 85 to 170 $\mathrm{mg} \cdot \mathrm{dl}^{-1}$. Chinese high values are near American low values. Similar relationships exist for the levels of plasma LDL cholesterol and the all-important ratio of total/HDL cholesterol. These very low plasma cholesterol levels in China are not surprising consider ing the very low intakes of dietary fat and animal protein. Plasma triglycerides also are significantly lower in China. These indicators of lipid status become more interesting when combined with indicators of protein status, namely plasma albumin, which is highly cor related with total cancer and coronary heart disease, and plasma urea nitrogen, which is highly correlated with animal protein intake. These latter univariate associations. and others, suggested that an increase in animal protein intake could be an important risk factor for the emergence of Western-type diseases in China. Further anal ysis of this effect has been undertaken using a multifactorial statisti cal method introduced to our group by Q. Yinsheng. A very highly significant relationship between indicators of protein status and mortality rates for Western-type diseases was observed. In another analysis with similar implications, there were statistically significant direct associations between several cancers and plasma choles terol, which was directly correlated. in turn, with total fat intake $(\mathrm{P}$ $<0.01)$ and meat consumption $(P<0.01)$. The lower circulating levels of beta carotene is consistent with the report of Yang et al. (1984) and is quite interesting in view of the greater consumption of this nutrient. The lower body size and body mass (i.e., overweight) of adults is particularly interesting in light of the higher level of energy intake (Table 1).

Briefly, these data especially when considered comprehensive ly. indicate that risk for Western-type diseases is increased with increasing intakes of animal foods. particularly when these associations are considered in reference to animal protein intake. What makes these associations particularly noteworthy is the fact that animal protein intake in China is already quite low when compared with Western intakes. Except for a few nomadic peoples living in the far north of China. the intake of animal protein (both fish and non-fish) only comprises from $0 \%$ to $15 \%$ to $20 \%$ of total protein intake. If chronic degenerative disease risk can be demonstrated for levels of animal protein intake this low, one cannot help but wonder what might be the risk that would be predicted for levels of animal protein intake in countries like the United States. That so much em phasis has been given to dietary fat in international correlation studies of diet and chronic degenerative diseases has overshadowed al most identical correlations with protein intake (National Academy of Sciences. 1982).

Therefore, I propose the hypothesis that the higher mortality rates for so-called diseases of affluence in the United States are ac counted for by the much greater consumption of animal products and not just the higher levels of saturated fat in these products that account for this effect. I suggest that the animal product effect is much more broadly based, almost certainly going beyond even fat and protein considerations. Thus, I would expand the hypothesis to state that, for each nutrient intake or each indicator of nutrient status that is strongly related to animal food intake, the only biologically plausible associations between these nutritional features and disease risk are those that are directly correlated. In other words, when risk for these diseases is affected nutritionally, an increased intake of animal foods, along with a series of companion nutrient intake changes. can only elevate risk. perhaps modestly or even negligibly for some individuals. perhaps substantially for others. Of course, it should be recognized that. as animal food intake goes up, plant food consumption goes down. When the nutritional properties of plant foods are examined in turn. the preponderance of evidence suggests a large variety of protective constituents.
This represents only the briefest sketch of the relationships between nutrition and the various diseases of affluence as presently existing in China; more sophisticated analyses of the data are presently underway.

If this analysis proves to be correct, there are many implications for policy development in the arenas of food. nutrition, agriculture, and health in developing countries such as China, including two that I will mention very briefly.

The notion that the production of livestock for human food should not be encouraged for nutritional reasons comes as welcome news, it seems to me, for those who are interested in the economics of this practice. I refer to the ample evidence indicating that the cost of producing a unit of animal food. such as protein for exam ple, is, according to some estimates, 18 to 20 times as much as producing the same unit of plant food (Glenn et al.. 1990). Thus, the arguments against encouraging the development of livestock indus tries in developing countries could be martialed on two fronts, one economic, the other nutritional.

The second implication derived from this hypothesis is the effect upon the further development of dietary guidelines in Western countries. I refer to the basis of the decisions to recommend reduc tion of dietary fat only down to $30 \%$ of calories, even when the evi dence indicates that it should still be lower. It has been my personal experience as a member of several deliberative groups on dietary guidelines that a substantial reason for not advocating still lower intakes of dietary fat is the ultimate necessity to decrease the consumption of animal products. against which there would be substantial political, economic, and even personal resistance for doing so. Often there is the implication that the intake of animal food in the United States should not be decreased because these foods contain certain important nutrients. such as highquality protein. If the evidence reviewed here is correct. then I would suggest that this appar ent disadvantage should be of little or no concern. That is. the high level of animal protein intake in the United States, in its own right, may be a significant determinant of the relatively high mortality rates for diseases of affluence that characterize disease trends in this country. Thus, in the further development of dietary guidelines in the United States. I would think that the recommendation to reduce dietary fat intake only to $30 \%$ of calories or so can be made more aggressive and more broadly based, without concern for the reduced level of animal protein intake.

In conclusion, there ought to be a very promising future for industries involved in the production of plant foods useful for human consumption-in all countries, rich and poor, East and West

\section{Literature Cited}

Chen, J., T.C. Campbell, R. Peto. and J. Li. 1990. A preliminary study of dietary. lifestyle and mortality characteristics of 65 rural populations in The People's Republic of China. Univ. of Oxford Press, Cornell Univ. Press. China People's Medical Publishing House (In press).

Federation of American Societies for Experimental Biology. 1987. Physiological effects and health consequence of dietary fiber. Center for Food Safety and Applied Nutrition. Food and Drug Administration, Department of Health and Human Services. Washington. D.C.

National Academy of Sciences. 1982. Diet, nutrition and cancer. National Academy Press. Washington. D.C.

O'Connor. T.P. and T.C. Campbell. 1986. Dietary guidelines, p. 731-771. In: C. Ip. D.F. Bin, C. Mettlin. and A.E. Rogers (eds.). Dietary fat and cancer, Liss. New York.

Pennington, J.A.T. 1983. Revision of the total diet study food list and diets. J. Amer. Diet. Assn. 82:166-173.

Yang, C.S., Y. Sun. Q. Yang, K.W. Miller. C. Li. S.F. Zheng. A.G. Ershow, W.J. Blot. and J. Li. 1984. Vitamin A and other deficiencies in Linxian. a high esophageal cancer incidence area in northern China. J. Nad. Cancer Inst 73:1449-1453. 